\title{
Radio-over-Fiber DSB-to-SSB Conversion Using Period-One Dynamics of Semiconductor Lasers
}

\author{
Yu-Han Hung ${ }^{1}$, Tzu-Yu Chen ${ }^{1}$, Sheng-Kwang Hwang ${ }^{1,2, *}$ \\ ${ }^{1}$ Department of Photonics, National Cheng Kung University, Tainan, Taiwan \\ ${ }^{2}$ Advanced Optoelectronic Technology Center, National Cheng Kung University, Tainan, Taiwan \\ *skhwang@mail.ncku.edu.tw
}

\begin{abstract}
Direct or external modulation scheme typically adopted in radio-over-fiber systems generates optical doublesideband modulation (DSB) signals. However, due to the microwave fading effect, optical single-sideband modulation (SSB) signals are preferred, therefore requiring DSB-to-SSB conversion. In this study, period-one nonlinear dynamics of semiconductor lasers is investigated to conduct such conversion for microwave frequency of more than $100 \mathrm{GHz}$. Only a typical laser is required and system self-tunability to the adjustment in microwave frequency is feasible.
\end{abstract}

\section{INTRODUCTION}

Radio-over-fiber has attracted much attention for wireless access networks, which distributes data-encoded microwave subcarriers over fibers to remote base stations. Direct or external modulation of semiconductor lasers is the simplest scheme to generate microwave subcarriers with high spectral purity. Optical doublesideband (DSB) modulation signals, however, are typically generated, leading to microwave fading over fiber distribution. To minimize the fading effect, optical single-sideband (SSB) modulation signals are preferred. Therefore, various DSB-to-SSB conversion schemes have been proposed [1-3]. In this study, we demonstrate the application of period-one (P1) nonlinear dynamics in semiconductor lasers for such conversion. Figure 1 shows the proposed system consisting of a typical single-mode semiconductor laser. Under continuous-wave (CW) optical injection with proper power and frequency, the laser intensity can exhibit high-frequency single-period oscillation at $f_{0}$, which is commonly referred to as $\mathrm{P} 1$ nonlinear dynamics. The optical spectrum consists of a regeneration of the injection and two oscillation sidebands equally separated away from the regeneration by $f_{0}$ with asymmetric intensity. Such an asymmetric feature is applied in this study for the DSB-to-SSB conversion if the input signal is microwave modulated with a DSB feature.

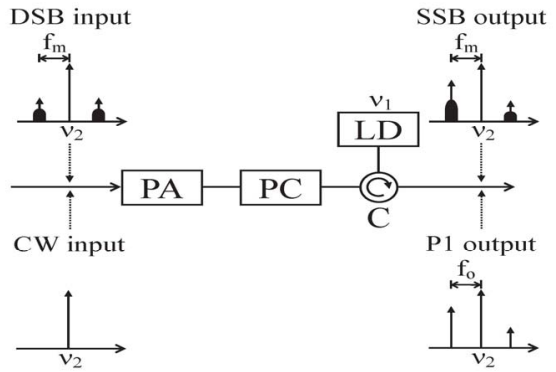

Fig. 1: Schematic of the proposed system and of the spectra for CW input and P1 output (lower row) and DSB input and SSB output (upper row). PA, power adjuster consisting of an optical amplifier or/and an attenuator; PC, polarization controller; C, circulator; LD, laser diode.

\section{NUMERICAL MODEL}

In this study, investigation is conducted through numerical calculation, and a single-mode model of a semiconductor laser subject to external optical injection is considered [3-5]

$$
\begin{aligned}
\frac{d a}{d t}= & \frac{1}{2}\left[\frac{\gamma_{c} \gamma_{n}}{\gamma_{s} \widetilde{J}} \widetilde{n}-\gamma_{p}\left(2 a+a^{2}\right)\right](1+a)+F_{a} \\
& +\xi_{i} \gamma_{c}\left[1+m\left(1+s(t) \cos \omega_{m} t\right)\right] \cos \left(\Omega_{i} t+\phi\right) \\
\frac{d \phi}{d t}= & -\frac{b}{2}\left[\frac{\gamma_{c} \gamma_{n}}{\gamma_{s} \widetilde{J}} \widetilde{n}-\gamma_{p}\left(2 a+a^{2}\right)\right]+\frac{F_{\phi}}{1+a} \\
& -\frac{\xi_{i} \gamma_{c}}{1+a}\left[1+m\left(1+s(t) \cos \omega_{m} t\right)\right] \sin \left(\Omega_{i} t+\phi\right) \\
\frac{d \widetilde{n}}{d t}= & -\gamma_{s} \widetilde{n}-\gamma_{n}(1+a)^{2} \widetilde{n}-\gamma_{s} \widetilde{J}\left(2 a+a^{2}\right) \\
& +\frac{\gamma_{s} \gamma_{p}}{\gamma_{c}} \widetilde{J}\left(2 a+a^{2}\right)(1+a)^{2}
\end{aligned}
$$

Here, $a$ is the normalized field amplitude of the injected laser, $\phi$ is the phase difference between the injection field and the injected laser, and $\tilde{n}$ is the normalized carrier density of the injected laser. The laser intrinsic parameters $\gamma_{c}, \gamma_{s}, \gamma_{n}, \gamma_{p}$, and $b$ are the cavity decay rate, spontaneous carrier decay rate, differential carrier decay rate, nonlinear carrier decay rate, and linewidth enhancement factor, respectively. The normalized, dimensionless bias current parameter $\widetilde{J}$ is the ratio between the amount of bias current above the threshold and the threshold bias level of the injected laser. The dimensionless injection parameter $\xi_{i}$ is proportional to the ratio of the electric fields between the injection signal and the free-running injected laser. The detuning frequency $f_{i}=\Omega_{i} / 2 \pi$ is the frequency offset of the injection field from the free-running frequency of the injected laser. The noise-source parameters, $F_{a}$ and $F_{\phi}$, are characterized by a spontaneous emission rate. The microwave carried by the injection signal is described in Eqs. (1) and (2), where $m$ is the microwave modulation index, $f_{m}=\omega_{m} / 2 \pi$ the microwave modulation frequency, and $s(t)$ the information. A second-order Runge-Kutta method is used to solve Eqs. (1)-(3) together with experimentally measured laser parameters [3-5], where $\widetilde{J}=1.222 \quad, \quad \gamma_{c}=5.36 \times 10^{11} \mathrm{~s}^{-1} \quad, \quad \gamma_{s}=5.96 \times 10^{9} \mathrm{~s}^{-1}$, $\gamma_{n}=7.53 \times 10^{9} \mathrm{~s}^{-1}, \gamma_{p}=1.91 \times 10^{10} \mathrm{~s}^{-1}$, and $b=3.2$. 


\section{RESULTS}

Figure 2(a) shows the optical spectrum of the injected laser subject to a $\mathrm{CW}$ optical input at $\left(\xi_{\mathrm{i}}, f_{\mathrm{i}}\right)=(0.4,57$ $\mathrm{GHz}$ ) under the period-one dynamics (blue curve). For comparison, the spectra of the injected laser under freerunning (gray curve) and the $\mathrm{CW}$ input (red curve) are also shown. A regeneration of the $\mathrm{CW}$ input appears due to the injection pulling effect. Equally separated oscillation sidebands from the regeneration by $f_{0}=60$ $\mathrm{GHz}$ also emerge, where the lower sideband is stronger by $35 \mathrm{~dB}$ compared to the upper one, resulting from the resonance enhancement of the red-shifted laser cavity caused by the injection [3-5]. Taking advantage of such sideband asymmetry enables the proposed DSB-to-SSB conversion, as Fig. 2(b) shows. Now the input optical signal is microwave-modulated at $f_{\mathrm{m}}=f_{0}=60 \mathrm{GHz}$, leading to a DSB feature (red curve) with the optical carrier 26-dB stronger than both modulation sidebands. Under the same $\left(\xi_{\mathrm{i}}, f_{\mathrm{i}}\right)$, the injected laser emits a nearly SSB output at exactly $f_{\mathrm{m}}$ (blue curve), where the lower modulation sideband is now stronger by $37 \mathrm{~dB}$ compared to the upper one. Figure 3 shows the data quality before and after the conversion (open and closed triangles), where a bit-error ratio (BER) down to $10^{-12}$ and a clear eye diagram are observed. This shows that the proposed system regenerates microwave features of an optical DSB input while converting its optical feature into SSB. To achieve the conversion of a DSB input at a different $f_{\mathrm{m}}, \xi_{\mathrm{i}}$ and/or $f_{\mathrm{i}}$ can be adjusted for a specific $f_{0}=f_{\mathrm{m}}$, up to at least $100 \mathrm{GHz}$, with a similar SSB output feature.

In fact, the system works even if $f_{\mathrm{m}} \neq f_{0}$. Under the same $\left(\xi_{\mathrm{i}}, f_{\mathrm{i}}\right)$ where $f_{0}=60 \mathrm{GHz}$, Fig. 2(c) shows a similar SSB output with $f_{\mathrm{m}}=64 \mathrm{GHz}$ (blue curve) for a DSB input at $f_{\mathrm{m}}=64 \mathrm{GHz}$ (red curve). This implies that the modulation sidebands lock P1 oscillation sidebands. Similar BER behavior is observed for this operating
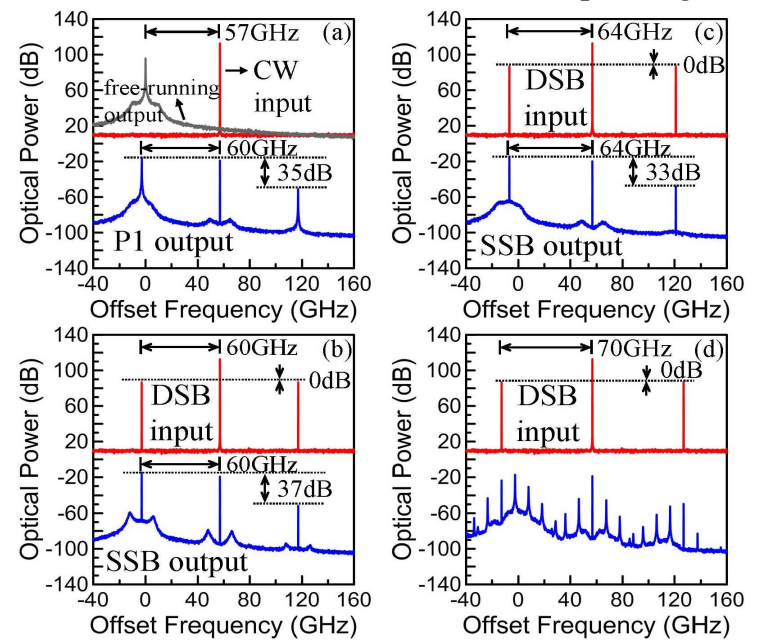

Fig. 2: Optical spectra of CW and DSB inputs (red curves), P1 and SSB outputs (blue curves), and free-running output (gray curve). For visibility, blue curves are shifted down with respect to red curves. Xaxes are relative to the free-running frequency of the injected laser.
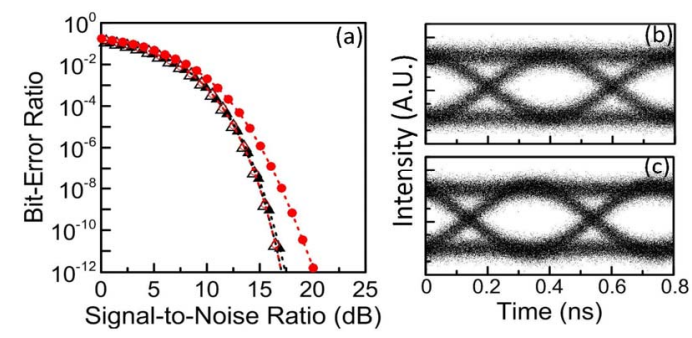

Fig. 3: (a) Bit-error ratio in terms of signal-to-noise ratio. Open and closed triangles for DSB input and SSB output, respectively, at $\left(\xi_{\mathrm{i}}, f_{\mathrm{i}}, f_{\mathrm{m}}\right)$ $=(0.4,57 \mathrm{GHz}, 60 \mathrm{GHz})$. Open and closed circles for DSB input and SSB output, respectively, at $\left(\xi_{\mathrm{i}}, f_{\mathrm{i}}, f_{\mathrm{m}}\right)=(0.4,57 \mathrm{GHz}, 64 \mathrm{GHz})$. All bit rates are fixed at $2.5 \mathrm{~Gb} / \mathrm{s}$. (b)(c) Eye-diagrams for open and closed triangles in (a), respectively, at BER $=10^{-9}$.

condition (open and closed circles). The system regenerates the DSB input while converting it with an SSB feature even if $f_{\mathrm{m}}$ varies from 54 to $65 \mathrm{GHz}$ under the present $\left(\xi_{\mathrm{i}}, f_{\mathrm{i}}\right)$, showing the system self-tunability to $f_{\mathrm{m}}$ adjustment without the change of the operating condition. As Fig. 2(d) shows, when $f_{\mathrm{m}}$ varies out of this locking range (red curve), unlocking between sidebands produces a nonlinear wave-mixing output (blue curve).

\section{CONCLUSION}

Period-one nonlinear dynamics of semiconductor lasers is demonstrated for DSB-to-SSB conversion. The proposed scheme depends solely on the property of input signals for a given laser, and thus only a typical laser is required as the main conversion unit. Conversion can be achieved for an operating microwave frequency of more than $100 \mathrm{GHz}$. System self-tunability to the adjustment in the operating microwave frequency is feasible. Numerical observations presented in this study have recently been verified by our preliminary experimental work.

\section{ACKNOWLEDGMENT}

This work is supported by National Science Council of Taiwan under Contract NSC99-2112-M-006-013-MY3.

\section{REFERENCES}

[1] G.H. Smith, D. Novak, and Z. Ahmed, "Overcoming chromatic-dispersion effects in fiber-wireless systems incorporating external modulators," IEEE Trans. on Microwave Theory Tech., vol. 45, pp. 1410-1415, 1997.

[2] A. Kaszubowska, P. Anandarajah, and L.P. Barry, "Multifunctional operation of a fiber Bragg grating in a $\mathrm{WDM} / \mathrm{SCM}$ radio over fiber distribution system," IEEE Photon. Technol. Lett., vol. 16, pp. 605-607, 2004.

[3] S.K. Hwang, S.C. Chan, S.C. Hsieh, and C.Y. Li, "Photonic microwave generation and transmission using direct modulation of stably injection-locked semiconductor lasers," Opt. Commun., vol. 284, pp. 3581-3589, 2011.

[4] S.K. Hwang and D.H. Liang, "Effects of linewidth enhancement factor on period-one oscillations of optically injected semiconductor lasers," Apply. Phys. Lett., vol. 89, pp. 061120, 2006.

[5] S.K. Hwang, H.F. Chen, and C.Y. Lin, "All-optical frequency conversion using nonlinear dynamics of semiconductor lasers," Opt. Lett., vol. 34, pp. 812-814, 2009. 